\title{
STEADY SLIDING OF A CIRCULAR CYLINDER OVER A DISSIMILAR THERMALLY CONDUCTING HALF-PLANE
}

\author{
D. A. Hills and J. R. Barber \\ Department of Engineering Science, Parks Road, Oxford, U.K. and Department of Mechanical Engineering \\ and Applied Mechanics, University of Michigan, Ann Arbor, MI48109, U.S.A.
}

(Received 25 March 1986; and in revised form 25 May 1986)

\begin{abstract}
The problem of a thermally conducting cylinder sliding steadily over the surface of an elastic half-plane with different thermal properties was analyzed. Appropriate Green's functions are used to reduce the problem to an integral equation which is solved numerically. Thermoelastic distortion due to frictional heating causes the contact patch to be smaller than that predicted for isothermal (Hertzian) contact although solutions assuming a single, perfectly conducting contact patch are not found for all values of parameters.
\end{abstract}

\section{INTRODUCTION}

Rubbing contact between convex bodies occurs frequently in engineering, in particular in certain types of brakes, in sliding electrical contacts and in wear tests. It has been conventional in analyzing these configurations to ignore the effect of thermal distortion produced by the heat generated, and to utilize Hertzian contact theory. However recent work by Barber and others (see Johnson [1]) has shown that because of thermoelastic coupling between the distorted profile and heat generation at the interface, very pronounced differences from isothermal contact are found, with much higher contact pressures. In particular, Barber [2] has shown that steady sliding of a conducting cylinder over an insulating half-plane (such that the contact is stationary in the conducting body) always results in a contact width which is narrower than that obtained in the isothermal problem. As the contact load is increased whilst the sliding speed is kept constant, the contact width asymptotically approaches a constant value which it cannot exceed. This is in contrast to the isothermal problem, where the contact size increases indefinitely with the applied load, within the other limits prescribed for Hertzian contact. It is the principal object of this paper to relax the assumption that the cylindrical contacting body is non-conductive, and investigate the effects of this generalization on the contact behaviour.

The more general case of two surfaces sliding together such that the contact patch is moving with respect to each body may be formulated by a similar technique. However, this serves to introduce further parameters into the argument, and hence for the present the particular case of one body being stationary is considered. This has the added advantage that comparison with an exact solution may be made.

\section{FORMULATION}

The geometry of the contacting pair is shown in Fig. 1. It is assumed that the cylinder is rotating with a peripheral velocity $v$, and that the region of contact is spatially fixed with

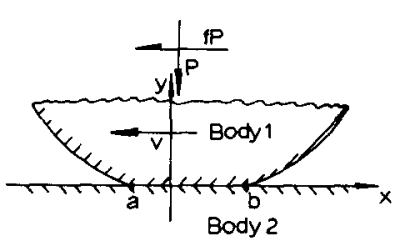

FIG. 1. Geometry of contact. 
respect to the lower plane. The elastic and thermal properties of the cylinder and the halfplane will generally be different and will be distinguished by the subscripts 1,2 , respectively.

First, we write down the $y$-direction displacement of body 1 at point $(x, 0)$ due to a line heat source of magnitude $q_{1}$ at point $(\xi, 0)$, travelling at constant speed $v$ which is

$$
\begin{array}{ll}
u_{\mathrm{thl}}=2 \frac{\delta_{1} k_{1}}{v} \exp \left\{-\frac{v(x-\xi)}{2 k_{1}}\right\} I_{0}\left\{\frac{v(x-\xi)}{2 k_{1}}\right\} & x>\xi \\
u_{\mathrm{th} 1}=2 \frac{\delta_{1} k_{1}}{v}, & x<\xi,
\end{array}
$$

where $\delta, k$ are, respectively, the thermal distortivity and diffusivity, and $I_{0}$ is a modified Bessel function (Barber [3]). It will be convenient to cast the problem in terms of the derivative of the normal displacement with respect to $x$, whereupon equations (1) become

$$
\frac{\mathrm{d} u_{\mathrm{th} 1}}{\mathrm{~d} x}=-\delta_{1} q_{1}(\xi) N(x-\xi) H(x-\xi),
$$

where $N(s)=\exp \left(-v s / 2 k_{1}\right)\left[I_{0}\left(-v s / 2 k_{1}\right)-I_{1}\left(v s / 2 k_{1}\right)\right]$ and $H(s)$ is Heaviside's step function.

Similarly, the slope of the surface of body 2 at point $x$ due to a static line heat source of magnitude $q_{2}$, located at $\xi$, is given by (Barber [4])

$$
\frac{\mathrm{d} u_{\mathrm{th} 2}}{\mathrm{~d} x}=\frac{\delta_{2} q_{2}(\xi) \operatorname{sgn}(x-\xi)}{2} .
$$

In addition, the surface displacements of the surfaces due to interfacial tractions are

$$
\begin{aligned}
\frac{\mathrm{d} u_{\mathrm{el} 1}}{\mathrm{~d} x} & =\frac{1-v_{1}}{\mu_{1} \pi} \int_{a}^{b} \frac{p(\xi) \mathrm{d} \xi}{x-\xi}-\frac{1-2 v_{1}}{2 \mu_{1}} t(x) \\
\frac{\mathrm{d} u_{\mathrm{el} 2}}{\mathrm{~d} x} & =-\frac{1-v_{2}}{\mu_{2} \pi} \int_{a}^{b} \frac{p(\xi) \mathrm{d} \xi}{x-\xi}-\frac{1-2 v_{2}}{2 \mu_{2}} t(x),
\end{aligned}
$$

where $\mu, v$ are, respectively, the modulus of rigidity and Poisson's ratio, and $p(x), t(x)$ are, respectively, the normal and shear traction present on the free surface.

It should be noted that since the bodies are sliding the direct and shear tractions are related at every point by the coefficient of Coulomb friction $f$, i.e.

$$
t(x)=f p(x) \text {. }
$$

Furthermore the heat sources $q_{1}(x), q_{2}(x)$ result from frictional work done against the tractions $t(x)$ and hence

$$
q_{1}(x)+q_{2}(x)=-f p(x) v
$$

(Note that this expression does not preclude the presence of a net heat flux due to an impressed temperature gradient between the bodies.)

Lastly, it will be assumed that the curvature of the bodies in the neighbourhood of contact may adequately be represented by parabolae, so that the initial gap, $g_{0}(x)$ between the bodies satisfies the equation

$$
\frac{\mathrm{d} g_{0}}{\mathrm{~d} x}=-\frac{x}{R},
$$

where $R$ is the radius of the cylinder. After deformation the gap becomes

$$
g(x)=g_{0}(x)-u_{1}(x)+u_{2}(x)
$$

where $u_{1}, u_{2}$ are the normal displacements of the surface due to both thermoelastic and elastic effects, i.e. $u=u_{\mathrm{el}}+u_{\mathrm{th}}$. In the contact region $a<x<b$, however, $\mathrm{d} g / \mathrm{d} x=0$ is required and hence, differentiating (8) with respect to $x$ and using equations (2)-(4) and (7) the following is 
obtained

$$
\begin{aligned}
& \left\{\frac{1-v_{1}}{\mu_{1}}+\frac{1-v_{2}}{\mu_{2}}\right\} \frac{1}{\pi} \int_{a}^{b} \frac{p(\xi) \mathrm{d} \xi}{x-\xi}-\left\{\frac{1-2 v_{1}}{\mu_{1}}-\frac{1-2 v_{2}}{\mu_{2}}\right\} \frac{f p(x)}{2} \\
& +\frac{\delta_{2}}{2} \int_{a}^{b} q_{2}(\xi) \operatorname{sgn}(x-\xi) \mathrm{d} \xi+\delta_{1} \int_{a}^{b} q_{1}(\xi) N(x-\xi) H(x-\xi) \mathrm{d} \xi \\
& =-\frac{x}{R} \quad a<x<b .
\end{aligned}
$$

Now define the following

$$
q(x)=q_{1}(x)-q_{2}(x)
$$

and simplify the appearance of (9) by introducing the notation

$$
\begin{aligned}
\Delta & =\frac{1}{2}(b-a) \\
\sigma & =\frac{1}{2}(b+a) \\
\tilde{p}(r) & =\frac{p(r) R}{P} \\
\tilde{q}(r) & =\frac{q(r) R}{P f v} \\
\mathrm{Pe} & =\frac{v \Delta}{2 k_{2}} \quad \text { (the Peclet number) } \\
H & =\frac{k_{2} \delta_{2}}{\left(1-v_{1} / \mu_{1}\right)+\left(1-v_{2} / \mu_{2}\right)} \\
\beta & =\frac{\left(1-2 v_{1}\right) / \mu_{1}-\left(1-2 v_{2}\right) / \mu_{2}}{\left(1-v_{1}\right) / \mu_{1}+\left(1-v_{2}\right) / \mu_{2}} \quad \text { (Dundurs' constant). }
\end{aligned}
$$

It should also be noted that for the corresponding isothermal problem, the contact halfwidth, $\Delta$, and contact load, $P_{H}$, are related by (Timoshenko and Goodier [5])

$$
\Delta^{2}=\frac{4 P_{H} R}{\pi}\left\{\frac{1-v_{1}}{\mu_{1}}+\frac{1-v_{2}}{\mu_{2}}\right\}
$$

and hence, equation (9) may be re-written as

$$
\begin{gathered}
\int_{-1}^{+1}\left\{\frac{1}{\pi(s-r)}-\frac{f \mathrm{Pe} H}{4}\left[-\frac{\delta_{1}}{\delta_{2}} N(s-r)+\frac{1}{2} \operatorname{sgn}(s-r)\right]\right\} \tilde{p}(r) \mathrm{d} r-\frac{\beta f \tilde{p}(s)}{2} \\
+\frac{f \mathrm{Pe} H}{4} \int_{-1}^{+1}\left\{\frac{\delta_{1}}{\delta_{2}} N(s-r)-\frac{1}{2} \operatorname{sgn}(s-r)\right\} \tilde{q}(r) \mathrm{d} r=-\frac{4}{\pi}(\sigma / \Delta+s)\left[\frac{R}{\Delta}\right]\left[\frac{P_{H}}{P}\right], \\
-1 \leqslant s \leqslant 1 .
\end{gathered}
$$

This equation can be regarded as a singular integral equation of the second kind for $p(r)$ in terms of $q(r)$ and known functions. It is noted that only the first term in the kernel has a Cauchy singularity, and the other terms are regular. The free term $(\beta f \tilde{p}(s) / 2)$ corresponds physically to the coupling between the tangential traction and the vertical displacement and has been shown by Goodman [6] to have only a small influence on the result in frictional contact problems. Therefore, subsequently, this term will be omitted, with the effect that (12) is reduced to a single integral equation of the first kind. Further, if it is assumed that body 1 is rigid, terms involving $\left(1-v_{1}\right) / \mu_{1}$ become zero.

The interfacial contact pressure must be in equilibrium with the applied load $P$, and hence

$$
P=-\int_{a}^{b} p(\xi) \mathrm{d} \xi
$$


or in normalized variables

$$
-1=\frac{\Delta}{R} \int_{-1}^{+1} \tilde{p}(r) \mathrm{d} r .
$$

Equations $(12,13)$ describe the relationship between surface tractions, heat flux and the contact geometry. A second equation must now be developed which relates the heat fluxes $q$ to the surface temperatures of the contacting bodies. It is assumed that there is no resistance to heat flow in the contact region so that the corresponding points on either side of the interface are at the same temperature. This requires

$$
\frac{\partial T_{1}}{\partial x}=\frac{\partial T_{2}}{\partial x}
$$

where $T_{1}, T_{2}$ are the surface temperatures.

Body 1 experiences a distribution of moving heat sources. The surface temperature at a point $(x, 0)$ due to a line heat source $q_{1}$ currently located at $(\xi, 0)$ and moving at speed $c$ is given by Carslaw and Jaeger [7] as

$$
T_{1}(x)=\frac{q_{1}(\xi)}{\pi K_{1}} \exp \left[\frac{-v(x-\xi)}{2 k_{1}}\right] K_{0}\left[\frac{v(x-\xi)}{2 k_{1}}\right]
$$

where $K_{1}$ is the thermal conductivity, and $K_{0}$ is the modified Bessel function. Differentiating, and summing the effects of a heat source distributed over the interval $a<x<b$, it is found that

where

$$
\frac{\partial T_{1}}{\partial x}=\frac{-v}{2 \pi k_{1} K_{1}} \int_{a}^{b} M(x-\xi) q_{1}(\xi) \mathrm{d} \xi
$$

$$
M(s)=\exp \left[-\frac{v s}{2 k_{1}}\right]\left\{K_{0}\left[\frac{v s}{2 k_{1}}\right]+K_{1}\left[\frac{v s}{2 k_{1}}\right]\right\} .
$$

Body 2 experiences a fixed heat source which gives rise to a surface temperature distribution (Carslaw and Jaeger [7])

$$
\frac{\partial T_{2}}{\partial x}=-\frac{1}{\pi K_{2}} \int_{a}^{b} \frac{q_{2}(\xi)}{x-\xi} \mathrm{d} \xi .
$$

Substituting equations (16) and (17) into (14) and making use of (10) gives

$$
\begin{aligned}
& \int_{a}^{b}\left\{\frac{v}{2 k_{1}}\left[\frac{K_{2}}{K_{1}}\right] M(x-\xi)+\frac{1}{x-\xi}\right\} q(\xi) \mathrm{d} \xi \\
& -f i \int_{a}^{b}\left\{\frac{v}{2 k_{1}}\left[\frac{K_{2}}{K_{1}}\right] M(x-\xi)+\frac{1}{x-\xi}\right\} p(\xi) \mathrm{d} \xi=0
\end{aligned}
$$

or, in terms of dimensionless variables

$$
\begin{aligned}
& \int_{-1}^{+1}\left\{\operatorname{Pe}\left[\frac{k_{2} K_{2}}{k_{1} K_{1}}\right] M[\Delta(s-r)]+\frac{1}{s-r}\right\} \tilde{q}(r) \mathrm{d} r \\
& -\int_{-1}^{+1}\left\{\operatorname{Pe}\left[\frac{k_{2} K_{2}}{k_{1} K_{1}}\right] M[\Delta(s-r)]-\frac{1}{s-r}\right\} \tilde{p}(r) \mathrm{d} r=0 .
\end{aligned}
$$

Lastly, it is convenient to introduce a variable quantifying the apportioning of frictional heat between the two bodies. Defining

$$
\lambda=\frac{Q_{1}-Q_{2}}{Q_{1}+Q_{2}}
$$

where $Q_{i}$ is the total heat flux into body $i$, gives

$$
\lambda=\frac{\Delta}{R} \int_{-1}^{+1} \tilde{q}(r) \mathrm{d} r .
$$


Thus, $\hat{\lambda}=-1$ when all the frictional heat is conducted into the half-plane, $\lambda=1$ when it is all conducted into the cylinder, whilst $\lambda=0$ corresponds to equipartition. In the physical problem involving bodies of finite dimensions, the value of $\lambda$ will depend upon the thermal boundary conditions distant from the contact region. It is not necessarily restricted to the range $-1<\lambda<1$, since there may be a superposed heat flux due to an externally imposed temperature difference between the contacting bodies.

As written, both integrals in equation (19) contain Cauchy singularities. However, a new function $\tilde{q}^{\prime}(r)$ may be introduced, defined by

$$
\tilde{q}(r)=\tilde{p}(r) \eta+\tilde{q}^{\prime}(r)
$$

Substituting into equation (19) gives

$$
\begin{gathered}
\int_{-1}^{+1}\left\{\operatorname{Pe}\left[\frac{k_{2} K_{2}}{k_{1} K_{1}}\right] M[\Delta(s-r)]+\frac{1}{s-r}\right\} \tilde{q}^{\prime}(r) \mathrm{d} r-\int_{-1}^{+1}\left\{\operatorname{Pe}\left[\frac{k_{2} K_{2}}{k_{1} K_{1}}\right]\right. \\
\left.\times M[\Delta(s-r)](1-\eta)-\frac{(1+\eta)}{s-r}\right\} \tilde{p}(r) \mathrm{d} r=0 .
\end{gathered}
$$

For small arguments

$$
M[\Delta(s-r)] \approx \frac{1}{\operatorname{Pe}(s-r)}
$$

and thus the Cauchy singularity in the second integral of equation (22) may be avoided by setting

$$
\eta=\frac{k_{2} K_{2} / k_{1} K_{1}-1}{k_{2} K_{2} / k_{1} K_{1}+1}
$$

\section{DISCRETIZATION}

The simultaneous integral equations (12) and (19) cannot be solved analytically and, in view of the Cauchy terms present in both, Gauss-Chebyshev quadrature is most appropriate. It was anticipated that $\tilde{p}(r)$ will be bounded at the end points whilst $\tilde{q}^{\prime}(r)$ will be singular, and hence it was decided to represent the solutions as the product of fundamental solutions and bounded continuous functions $\phi(r), \psi(r)$, thus

$$
\begin{aligned}
& \tilde{p}(r)=\left(1-r^{2}\right)^{1 / 2} \phi(r) \\
& \tilde{q}^{\prime}(r)=\left(1-r^{2}\right)^{-1 / 2} \psi(r) .
\end{aligned}
$$

Hence, the discretized form of equation (12) is (Erdogan et al. [8]);

where

$$
\begin{aligned}
& \sum_{i=1}^{n} \frac{1-r_{i}^{2}}{n+1}\left[\frac{1}{s_{k}-r_{i}}+\pi \frac{f \mathrm{Pe} H}{4} G_{1}\left(s_{k}, r_{i}\right)\right] \frac{\phi\left(r_{i}\right)}{-\Lambda} \\
& +\sum_{i=1}^{n} \frac{\pi}{n} G_{2}\left(s_{k}, r\right) \frac{\psi(r)}{-\Lambda}=\frac{\sigma}{\Lambda}+s_{k} \\
& k=1 \ldots n+1,
\end{aligned}
$$

$$
\begin{aligned}
r_{i} & =\cos \left[\frac{\pi i}{n+1}\right] \\
s_{k} & =\cos \left[\frac{\pi}{2} \frac{2 k-1}{n+1}\right] \\
\Lambda & =\frac{4 R P_{\mathrm{H}}}{\pi \Delta P} \\
G_{1}(s, r) & =-\frac{\delta_{1}}{\delta_{2}} N(s-r)(1-\eta)+\frac{1}{2} \operatorname{sgn}(s-r)(1+\eta) \\
G_{2}(s, r) & =\frac{\delta_{1}}{\delta_{2}} N(s-r)-\frac{1}{2} \operatorname{sgn}(s-r)
\end{aligned}
$$


and equation (13) becomes

$$
-1=\frac{\Delta}{R} \frac{\pi}{n+1} \sum_{i=1}^{n}\left(1-r_{i}^{2}\right) \phi\left(r_{i}\right),
$$

i.e.

$$
\frac{P_{H}}{P}=-\frac{2}{(n+1) \Lambda} \sum_{i=1}^{n}\left(1-r_{i}^{2}\right) \phi\left(r_{i}\right)
$$

thereby defining the dimensionless load parameter, $P_{H} / P$.

Similarly, the discretized form of (22) is:

$$
\begin{aligned}
& \sum_{i=1}^{n} \frac{\pi}{n} G_{3}\left(s_{k}^{\prime}, r_{i}^{\prime}\right) \frac{\psi\left(r_{i}^{\prime}\right)}{-\Lambda}+\sum_{i=1}^{n} \frac{1-r^{2}}{n+1} \pi G_{4}\left(s_{k}^{\prime}, r\right) \frac{\phi(r)}{-\Lambda}=0 \\
& k=1 \ldots n-1,
\end{aligned}
$$

where

$$
\begin{aligned}
r_{i}^{\prime} & =\cos \left[\pi \frac{2 i-1}{2 n}\right] \\
s_{k}^{\prime} & =\cos \left[\frac{\pi k}{n}\right] \\
G_{3}(s, r) & =\operatorname{Pe}\left[\frac{k_{2}}{k_{1}}\right]^{2} M[\Delta(s-r)]+\frac{1}{s-r} \\
G_{4}(s, r) & =-\operatorname{Pe}\left[\frac{k_{2}}{k_{1}}\right]^{2}(M[\Delta(s-r)])(1-\eta)-\frac{1}{s-r}(1+\eta)
\end{aligned}
$$

and, lastly, equation (20) becomes

$$
\lambda=-\sum_{i=1}^{n} \frac{1}{n} \psi\left(r_{i}^{\prime}\right) / \sum_{i=1}^{n} \frac{1-r_{i}^{2}}{n+1} \phi\left(r_{i}^{\prime}\right)-\eta .
$$

The $2 n$ simultaneous linear algebraic equations given in equations (25) and (27) are sufficient to enable $\phi\left(r_{i}\right), \psi\left(r_{i}^{\prime}\right)$ to be found at the integration points, after which $P_{H} / P$ and $\lambda$ can be found from equations (26) and (28).

\section{VALIDITY OF SOLUTION}

The classical existence and uniqueness theorems of heat conduction and elasticity do not apply to thermoelastic contact problems with unilateral boundary conditions. Indeed, counter-examples have been discovered to both in steady-state problems (see Barber [4], Comninou and Dundurs [9]). There is therefore no guarantee that the stated problem will have a solution for all values of the physical parameters, nor that a solution when obtained will be physically reasonable.

It is therefore necessary to check the solution when obtained, to ensure that the inequalities of unilateral contact are satisfied, i.e. (a) that the interfacial traction in the contact region is non-tensile and (b) that the gap in the separation region is non-negative (so that there is no interpenetration of material).

Violations of type (a) are readily detected from the calculated values of $\tilde{p}$, but further analysis is required to determine the gap between the bodies, exterior to the contact region. Equations (1) and the integrated form of equation (3) were used to describe the effect of heat flux on surface displacement; and the integrated form of equations (4) to describe the effect of surface tractions. Their combined influence, neglecting the effect of shear tractions, is to produce a gap $g(x)$ given by;

$$
\begin{aligned}
g(x)= & \frac{x^{2}}{2 R}+\left\{\frac{1-v_{1}}{\mu_{1}}+\frac{1-v_{2}}{\mu_{2}}\right\} \frac{1}{\pi} \int_{a}^{b} p(\xi) \log \left[\frac{x-\xi}{\Delta}\right] \mathrm{d} \xi-\int_{a}^{b} 2 \frac{\delta_{1} k_{1}}{v_{1}} \exp \left\{-\frac{v(x-\xi)}{2 k_{1}}\right\} \\
& \times I_{0}\left\{\frac{v(x-\xi)}{2 k_{1}}\right\} q_{1}(\xi)-\frac{1}{2} \delta_{2}(x-\xi) q_{2}(\xi) d \xi+c_{1} \quad x>b
\end{aligned}
$$




$$
\begin{aligned}
g(x)= & \frac{x^{2}}{2 R}+\left\{\frac{1-v_{1}}{\mu_{1}}+\frac{1-v_{2}}{\mu_{2}}\right\} \frac{1}{\pi} \int_{a}^{b} p(\xi) \log \left[\frac{x-\xi}{\Delta}\right] \mathrm{d} \xi \\
& +\frac{1}{2} \delta_{2} \int_{a}^{b}(\xi-x) q_{2}(\xi) \mathrm{d} \xi+c_{2} \quad x<a,
\end{aligned}
$$

where the rigid body terms $c_{1}, c_{2}$ are adjusted so that $g(x)$ vanishes at $x=b, a$. Equations $(29,30)$ are normalized and discretized in the same manner as the principal equations $(9,18)$.

\section{RESULTS}

In practice, it is natural to regard $f, H, K_{2} / K_{1}, k_{2} / k_{1}, \delta_{2} / \delta_{1}$ as parameters, $P, v, \lambda$ as independent variables (the last controlled by adjusting the far field temperatures), and $\Delta, \sigma$ (i.e. Pe, $P_{H} / P$ ) as dependent variables. Whilst $f, H, K_{2} / K_{1}, k_{2} / k_{1}, \delta_{2} \delta_{1}$ remain parameters in this formulation it is more convenient to treat $a / R, b / R$ and $\mathrm{Pe}$ as independent variables, and to deduce values of $P_{H} / P, \lambda$ and $f H \mathrm{Pe}$ (note that the last quantity is equivalent to the dimensionless quantity designated $a \beta$ by Barber [2]). Further, to reduce the number of free variables to manageable proportions, set $\delta_{2} / \delta_{1}=1$ and $K_{2} / K_{1}=k_{2} / k_{1}$, which may be interpreted physically as assigning the same specific thermal capacity to the two bodies. The effect of $\lambda$ is of greater interest than the location of the contact patch $(\sigma / R)$ and hence in obtaining the results frequent adjustments of this quantity were made to enable convenient values of $\lambda$ to be yielded.

The system analyzed reduces to that treated by Barber [2] in the limit where $K_{1}$ tends to zero, i.e. where the cylinder is a non-conductor. Figure 2 compares this limiting solution with the present results for the case where $K_{2} / K_{1}=100$, so that the cylinder is a much poorer conductor than the half-plane. Iteration was used to maintain the value of $\lambda$ at -1 , i.e. to ensure that all the frictional heat flowed into the stationary body (though of course for any non-zero value of $K_{1}$, there will be local heat flow into the cylinder in parts of the contact region, balanced by regions of heat outflow). For a Peclet number of 5.0, convergence of the numerical scheme was obtained with $n=50$, which yields the largest matrix $(100 \times 100)$ which can conveniently be inverted.

In the limit, the solution should be independent of velocity except through the parameter $f H \mathrm{Pe}$. Therefore the calculations were repeated for $\mathrm{Pe}=10.0$ (see Fig. 2). Convergence on the limiting solution is good at larger values of $f H P e$, but less good at smaller values.

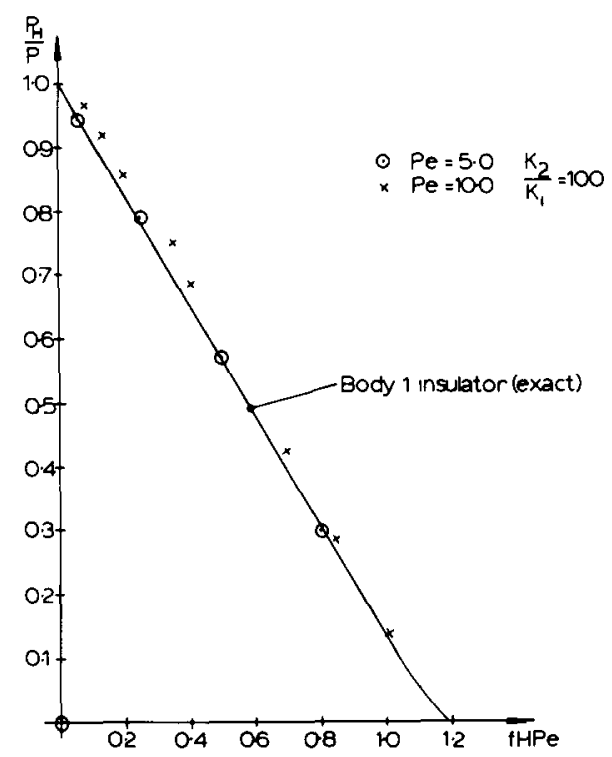

FIG. 2. Convergence of present solution on existing one for the case of a sliding insulated cylinder. 
It should be noted that these values of Peclet number are large in the context of heat conduction theory and correspond to situations where there is very little conduction of heat in the cylinder parallel to the direction of motion, because the period during which a specific point is in contact is very short in comparison with the heat induction transient. One consequence is that the Green's function for thermoelastic distortion in the cylinder [equation (1)] approaches closely to a step function, and its derivative [equation (2)] to a $\delta$ function. This necessitates the use of a large number of discretization points at large values of Peclet number, if adequate accuracy is to be obtained. This question is further discussed by Hills and Barber [10], in the solution of a related problem for a moving rigid punch.

The ratio $K_{2} / K_{1}$ was reduced to 10 , whilst $i$ was again maintained at -1 by iteration, giving the results shown in Fig. 3. The same general trend was observed, but the contact width is in each case larger than that obtained with an insulating cylinder, the difference increasing as the Peclet number is reduced. It is also found that $(\sigma / \Delta)$ increases with $f H \mathrm{Pe}$ and $\mathrm{Pe}$. In other words, the contact region becomes progressively displaced downstream around the cylinder as $f H \mathrm{Pe}$ (and hence the magnitude of the thermoelastic effects) increased.

A more striking conclusion is that some of the solutions obtained violate the physical inequalities, indicating that there is no solution of the type assumed in some ranges of the physical parameters. In particular, violations are obtained at high values of applied load. $P$. The dependence on speed is somewhat more complicated, since Pe appears both explicitly and in the combination $f H P e$ and the Peclet number also contains the contact semi-width. $\Delta$. which also appears in the definition of $P_{H}$. However, it seems that violations are more prevalent at lower values of speed $V$, where presumably thermoelastic effects in the cylinder become more significant. No attempt was made to obtain results for Peclet numbers in excess of 10 , in view of the accuracy problems discussed above and it proved impossible to obtain any solutions without violations for Peclet numbers less than unity.

The point at which violations start to occur is well-defined and is usually signalled by the occurrence of interpenetration just downstream of the trailing edge of the contact region. $x=a$, often with the simultaneous occurrence of tensile contact tractions near to the same transition. This behaviour is very similar to that demonstrated by Comninou and Dundurs [11] in steady-state thermoelastic contact when the heat flow is locally directed into the body with the smaller distortivity. In steady-state problems, this difficulty has been overcome by postulating the existence of a pressure dependent thermal contact resistance between the bodies (Barber [12]), but it is not clear whether such a device would be sufficient to guarantee

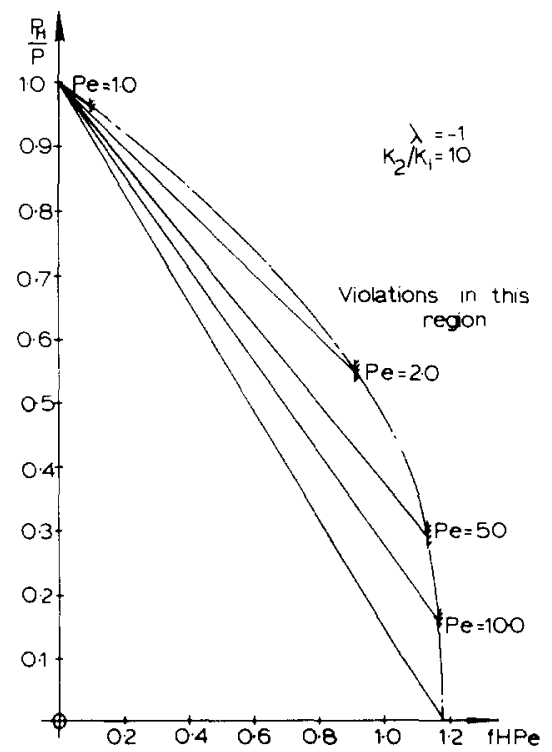

FiG. 3. The effect of sliding speed on contact patch size where net heat flux corresponds to all heat dissipating in the stationary body. 


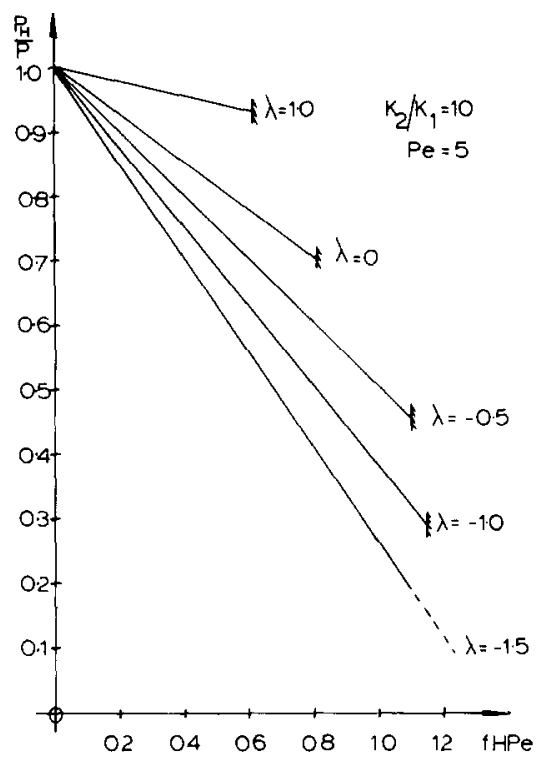

FIG. 4. The effect of partitioning of total heat generated on contact patch size.

existence in the present problem, or whether some different kind of configuration is to be anticipated, such as one involving several disconnected contact regions, or a cyclic behaviour of some kind.

Figure 4 shows the effect of varying $\lambda$ whilst keeping $K_{2} / K_{1}$ and Pe fixed at 10 and 5 , respectively. In interpreting this Figure, it is helpful to remember that if there were no thermoelastic effects, the load (for a given contact width and hence for a given value of $f H \mathrm{Pe}$ ) would always be equal to the Hertzian load, giving a horizontal line in Fig. 4. The fact that all the lines fall with increasing $f H P e$ indicates that the thermoelastic distortion always causes the contact width for a given load to be smaller than the Hertzian, but this effect is reduced as $\lambda$ increases and is relatively small for $\lambda=1$, when all the frictional heat is directed into the moving body. This argues that the thermoelastic distortion of the stationary half-plane is principally responsible for the modification of the Hertzian solution-a conclusion which seems reasonable in view of the fact that the moving body is continually presenting new 'isothermal' material to the contact region, so that there is relatively little opportunity for extensive thermal distortion to occur during a simple passage through the contact region.

Confirmation of this conclusion is presented in Fig. 5, which shows the load required to establish a given contact width over a wider range of $\lambda$ from -1 to 4 . For $\lambda>1$, there is a net heat flow out of the half-plane (which implies the imposition of a temperature difference between the distant boundaries of the bodies, with a corresponding superposed heat flux). In this range, the load $P$ is less than the Hertzian value, as might be anticipated, since the halfplane will contract locally to the contact region, making the bodies more conforming.

\section{CONCLUSIONS}

A solution has been given for the steady-state thermoelastic contact of an elastic cylinder sliding against a stationary elastic half-plane. The thermoelastic distortion due to frictional heating causes the contact region to be smaller than the Hertzian value and the results approach the previously published solution for a rigid, non-conducting cylinder at large values of the ratio of conductivities.

In general, the contact problem is dominated by the distortion of the stationary half-plane. When most of the heat flows into the moving cylinder, the contact width is much closer to the Hertzian and is less sensitive to variation of speed or coefficient of friction.

Physically acceptable solutions are not obtained in certain ranges of the parameters, indicating that either the boundary conditions are too idealized or the contact configuration 


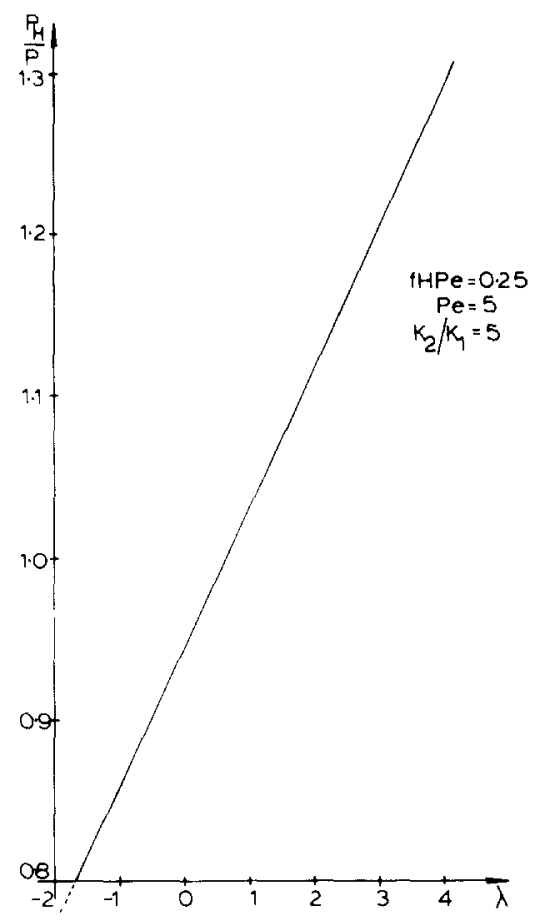

FIG. 5. Variation of partitioning of total heat with applied load.

differs from that assumed. The behaviour of the system in such ranges will be a topic of future investigations.

The most obvious application of this analysis is to the contact of composite brake blocks on steel railway-vehicle wheels. It might be anticipated that such configurations lead to the conduction of the majority of the heat into the wheel, and hence into the moving body. However, recent work by Barber et al. [13] indicates that the hot spots developed are stationary in the wheel and traverse the block. Thus, the present analysis, where we place an emphasis on the majority of the heat passing into the stationary body, is quite relevant to such cases.

Acknowledgements-The authors thank Alison for her great patience and perseverance in typing this manuscript. J. R. Barber gratefully acknowledges the support of the National Science Foundation under grant number MSM 8419324.

\section{REFERENCES}

1. K. L. Johnson, Contact Mechanics, Chap. 12. Cambridge University Press (1985).

2. J. R. BARBER, Some thermoelastic contact problems involving frictional heating. Q. J. appl. Math. 29, 1-13 (1976).

3. J. R. BARBER, Thermoelastic displacements and stresses due to a heat source moving over the surface of a halfplane. J. appl. Mech. 51, 636-640 (1984).

4. J. R. BARBer. Indentation of the semi-infinite solid by a hot sphere. Int. J. Mech. Sci. 15, 813-819 (1973).

5. S. P. Timoshenko and J. N. Goodier, Theory of Elasticity, 3rd edn, pp. 409-420, McGraw-Hill, Maidenhead, U.K. (1934).

6. L. E. Goodman, Contact stress analysis of normally loaded rough spheres. J. appl. Mech. 84, 515-522 (1962).

7. H. S. Carslaw and J. C. JaEger, Conduction of Heat in Solids, p. 267. Oxford University Press (1959).

8. F. ERdogAN, G. D. GuPTA and T. S. COOK, Numerical solution of singular integral equations. In Methods of Analysis and Solution of Crack Problems. Noordhoff, Leyden, New York (1973).

9. M. Comninou and J. Dundurs, On lack of uniqueness in heat conduction through a solid to solid interface. ASME J. Heat Transfer 102, 319-323 (1980).

10. D. A. HILLS and J. R. BARBER, Steady motion of an insulating rigid flat-ended punch over a thermally conducting half-plane. Wear 102, 15-22 (1985).

11. M. Comninou and J. Dundurs, On the Barber boundary conditions for thermoelastic contact. ASME J. appl. Mech. 42, 849-853 (1979).

12. J. R. BARBer, Contact problems involving a cooled punch. J. Elast. 8, 409-423 (1978).

13. J. R. Barber, T. W. Bealmond, J. R. WARInG and C. Pritchard, Implications of thermoelastic instability for the design of brakes. Trans. ASME J. Tribol. 107, 206-210 (1985). 